\title{
Carnets
}

Revue électronique d'études françaises de l'APEF

Deuxième série - 14 | 2018

Études de génétique théâtrale et littéraire

\section{Dynamiques de la réécriture dans l'œuvre de Didier-Georges Gabily : étude de la pièce Gibiers $d u$ temps}

\section{Séverine Leroy}

\section{(2) OpenEdition}

Journals

Édition électronique

URL : http://journals.openedition.org/carnets/8869

DOI : $10.4000 /$ carnets.8869

ISSN : 1646-7698

Éditeur

APEF

Référence électronique

Séverine Leroy, « Dynamiques de la réécriture dans l'œuvre de Didier-Georges Gabily : étude de la pièce Gibiers du temps », Carnets [En ligne], Deuxième série - 14 | 2018, mis en ligne le 30 novembre 2018, consulté le 19 avril 2019. URL : http://journals.openedition.org/carnets/8869 ; DOI : 10.4000/ carnets.8869

Ce document a été généré automatiquement le 19 avril 2019.

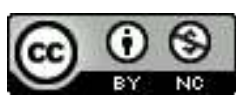

Carnets est mis à disposition selon les termes de la licence Creative Commons - Atribution - Pas d'utilisation commerciale 4.0 International. 


\title{
Dynamiques de la réécriture dans l'œuvre de Didier-Georges Gabily : étude de la pièce Gibiers du temps
}

\author{
Séverine Leroy
}

1 Les dynamiques de la réécriture parcourent la majeure partie des productions de l'auteur et metteur en scène français Didier-Georges Gabily (1955-1996). S'appuyant sur des hypotextes issus de genres semblables, avec Gibiers du temps (Gabily, 1995), Chimère et autres bestioles (Gabily, 1994), Zoologie (Gabily, inédit) et Contention (Gabily, 2008), pièces dans lesquelles il met en scène les personnages mythiques et littéraires (Phèdre, Thésée, (Edipe, Dom Juan, Le Prince et Hermiane) ou effectuant, au contraire, des réécritures inspirées de genres différents comme en atteste sa pièce TDM3 - Théâtre du Mépris (Gabily, 1996) basée sur l'Odyssée d'Homère (Homère, 1955), Le Mépris d'Alberto Moravia (Moravia, 1954) et Le Mépris de Jean-Luc Godard (Godard, 1963), les œuvres de Gabily n'ont de cesse d'interroger l'espace contemporain au prisme de ses héritages. Écrit entre 1994 et 1995, Gibiers du temps est un triptyque composé de trois époques: Thésée, Voix et Phèdre, fragments d'agonie. L'axe principal de la fable repose sur le retour de Thésée différé de plusieurs siècles et qui le fait arriver dans une mégapole occidentale de la fin $\mathrm{du} \mathrm{xx}^{\mathrm{e}}$ siècle. La tension dramaturgique repose en partie sur le décalage entre le héros, imprégné des valeurs de la Grèce antique, et celles de la société contemporaine. Le trajet de Thésée n'est autre que son acheminement vers le sacrifice ritualisé du héros mythique, qui prend ici la forme d'une mise à mort dans le cadre d'un banquet de clôture. De toutes les pièces de reprise de mythes écrites par Gabily, Gibiers du temps est celle où le choc entre le passé et le présent est le plus violent. C'est aussi celle où l'auteur s'est appliqué à construire une fable complexe invitant le lecteur et spectateur à de multiples questionnements relatifs aux héritages théâtraux et, plus largement, culturels et sociaux. Le choc entre passé et présent en passe d'abord par la distribution des personnages. Les personnages mythiques côtoient ici l'espace de la contemporanéité de l'auteur. Situés en différents points de connaissance du monde d'aujourd'hui, ceux-ci sont plus ou moins en décalage avec les codes et cette variabilité produit en elle-même du sens. 
2 Précédée d'une analyse génétique qui nous permettra de saisir l'importance de la pratique du théâtre dans l'écriture de l'auteur, nous procéderons ensuite à une analyse comparée de certains mythèmes afin de découvrir les relations qui s'opèrent entre les œuvres antérieures et la pièce de Gabily. Nous démontrerons ainsi les jeux littéraires de transposition et d'hyper-textualité tout en les inscrivant dans une réflexion critique sur la société contemporaine.

\section{Génétique de la pièce : un atelier de formation de l'acteur}

3 Le geste d'écriture de D.-G. Gabily s'inscrit pleinement dans le travail de recherche et de formation qu'il mène auprès des acteurs et qui est la source de son écriture. C'est dans ces ateliers que le formateur d'acteur - pas encore déclaré auteur et metteur en scène dans le milieu professionnel - conduit les apprentis à explorer les langues des auteurs tragique, baroque, classique et contemporain et à éprouver physiquement la diversité des poèmes tout autant que les questions qu'ils adressent à la pratique théâtrale. La pièce Gibiers $d u$ temps porte les traces de l'atelier Phèdre(s) et Hippolyte(s) mené en juillet 1990. Cette exploration réunissait les écritures du mythe de Phèdre chez Euripide, Sénèque, Garnier, Racine, Tsvetaïeva et Ritsos. Gabily voulait que ces différentes versions du mythe soient réunies en étant portées l'une après l'autre par les comédiens :

Donc s'enfiler (sans filet) six fois Phèdre, six fois Hippolyte. Six Phèdres et Hippolytes. Avec la participation (obligée et par ordre chronologique) d'Euripide, Sénèque, Garnier, Racine, Tsvetaïeva, Ritsos. Vouloir commencer à aboutir (?) quelque chose d'un travail entamé il y a plusieurs mois et qui se poursuivra - tant la matière (au sens premier du mot) est riche et d'usage multiple. (Gabily, 2003 : 24)

4 Cette note laisse à imaginer combien Gabily pensait son geste dans la perspective d'une généalogie d'auteurs et dans une conscience de leurs héritages. À travers l'atelier, les acteurs éprouvaient les glissements auquel le mythe est soumis. Le geste artistique témoigne de l'engagement de l'auteur dans un mouvement de filiation, autre dimension mémorielle qui fait à la fois «brèche et suture » selon les termes de Paul Ricœur (2000: 514). Dans ses notes de travail, Gabily exprime son projet en ces termes:

Nous visitons six maisons. Les maisons sont rarement vides : traces de mobiliers (d'immobiliers?) anciens, des voix, des corps, voire des idéologies du "mettre en scène", du "comment penser à, le rôle", etc. Nous confrontons scènes à scènes les bribes d'une histoire plurielle, pourtant toujours la même, les strates d'une histoire disparate et commune du "comment s'établit et se vérifie le principe d'exclusion de la cité". Par exemple. Scènes à scènes, textes à textes. Pas de réponse unifiante, lénifiante, mais des indications. Selon le temps de la réécriture du mythe. Selon l'usage et selon les modes qui y présidèrent. Ainsi, nous aimions la "limpidité" racinienne, attestée par les maîtres et quelques petits siècles de soi-disant classicisme français; il nous arrive d'y voir des pauvretés insanes, des abaissements devant les bienséances qui disent le, qui parlent $d u$ poids de la peur et des refoulements dans une société donnée, à une époque donnée; notre amour change de lieu. Nous aimons chez Racine ce qui se tait, comme chez Garnier nous avons appris à aimer ce qui se dit en trop, c'est-à-dire humainement; oui nous trouvons chez Garnier, l'or de la langue boueuse et grasse, enracinée jusqu'à l'outrance, la redite des souffrances des hommes. (Gabily, $2003: 24-25$ )

5 Comment la langue évolue-t-elle ? Comment le théâtre évolue-t-il ? Comment dire le mythe en différents moments de l'histoire des hommes? De cette traversée dans six 
versions différentes du mythe de Phèdre et Hippolyte, Gibiers du temps en porte ponctuellement les traces, mais la pièce manifeste essentiellement une grande démarcation d'avec ses prédécesseurs. Toutefois, la version d'Euripide est celle à laquelle Gabily a le plus emprunté : «Redire encore ici qu'il s'agit - malgré les (nombreuses) restrictions d'usage - d'une adaptation de l'Hippolyte d'Euripide.» (Gabily, 2003: 106) Quels emprunts ou quelles influences peut-on déceler chez Gabily? En quoi sa fable se démarque-t-elle des précédentes versions sur lesquelles le Groupe $\mathrm{T}^{\prime} \mathrm{Chan}^{\prime} \mathrm{G}^{1}$ a travaillé ? Si l'on s'appuie sur la conception que Lévi-Strauss propose du mythe en le décomposant en mythèmes, lesquels seront alors opératoires dans le mythe qui nous occupe?

Il ne semble pas incohérent de considérer que les premiers éléments nécessaires à la permanence de ce mythe sont la dévoration du désir de Phèdre pour Hippolyte et le lien de filiation qui unit ces deux personnages jusque dans l'interdit. De cet amour inassouvi, découle la mort des personnages provoquée par la húbris de Phèdre qui, par son mensonge, provoque la condamnation de l'innocent Hippolyte par son propre père: Thésée. Le nœud mythique donne à la tragédie sa possibilité d'être, c'est-à-dire qu'il y a faute et combat stérile contre cette faute, crime et combat stérile contre ce crime. Dans les cinq versions théâtrales ${ }^{2}$ traversées par le Groupe T'Chan'G en 1990, les structures dramaturgiques d'Euripide, Sénèque et Garnier sont assez proches. Les variables se situent essentiellement dans le rôle de la nourrice, la modalité de l'aveu de Phèdre à Hippolyte, la façon dont elle se donne la mort et dans la confrontation de Phèdre à Thésée. Racine a quant à lui écrit une pièce répondant aux règles de la poétique d'Aristote reprise en son temps par l'Abbé d'Aubignac et a modifié certains éléments du mythe pour servir sa pièce et l'adapter selon les règles de la bienséance et de la vraisemblance. On notera notamment la liberté prise par l'auteur classique à l'égard de la haine que le jeune homme conçoit pour les femmes en attribuant à ce dernier un amour impossible pour la jeune Aricie, sœur des Pallantides tués par Thésée. Racine introduit ainsi un personnage qui n'appartient pas au mythe et transforme le fondement de la vengeance de Phèdre, désormais animée par le sentiment de la jalousie plutôt que par le dépit amoureux. À travers Phèdre, Hippolyte et Aricie, Racine étend la question du désir à d'autres personnages. Gabily en fait de même dans Gibiers du temps. Racine fait également intervenir une dimension politique car, avec l'annonce de la mort de Thésée, s'ensuivent des tractations relatives au trône et à la succession du roi par Phèdre ou son fils, Hippolyte ou Aricie. Cet aspect politique se retrouve chez Gabily où règnent les fils de Thésée et de Phèdre : Acamas et Démophon. Dans la contemporanéité de Gibiers du temps, le pouvoir de ces deux personnages mythiques n'est plus de l'ordre du politique mais de l'économique dans ce qu'il a de plus perverti. En effet, les fils de Phèdre et de Thésée sont des personnages inspirés des parrains de la mafia que le cinéma et les séries télévisées érigent en héros des fictions modernes : trafiquants de drogues, producteurs de films pornographiques, esclavagistes et oppresseurs des femmes. Les activités de la descendance exposent la misère de notre monde et inscrivent la pièce dans une critique féroce du système d'exclusion et de lutte que continuent de se donner les hommes et les femmes. Par-delà ces différences, les auteurs se démarquent les uns des autres au gré de leur langue et de l'importance qu'ils accordent à certains aspects du récit plus qu'à d'autres. De son côté, Gabily prélève deux mythèmes que sont le désir monstrueux de Phèdre et la volonté de se donner la mort. Autour de ces deux éléments fondateurs, il agrège des personnages, des lieux, des actions qui réduisent l'unité d'action initiale à une 
masse composite et fourmillante, à un agrégat qui déplace la passion de Phèdre vers la tragédie du désir et concerne alors bien d'autres personnages :

Donc, Thésée aurait été empêché de revenir en d'autres temps où on avait besoin de lui pour borner l'inceste, c'est-à-dire le désir au-delà. Et qu'en serait-il advenu alors de Phèdre et d'Hippolyte ? Tous Phèdre(s), tous Hippolyte(s), peut-être : un rêve, un songe, un cauchemar inracontable que vient battre en brèche l'efficace et prégnante et apprenante réalité d'aujourd'hui. Ou plutôt, les réalités. Et parmi ces réalités, ce qu'il en est du commerce du désir. (...) Du bas (ceux d'en bas, les délaissés) en haut (ceux d'en haut, la descendance des puissants, les encore plus puissants maintenant que les dieux sont morts). Commerce du désir, rien d'autre, sur cette fraction de planète par nous habitée. Commerce, assouvissement, commerce, faillite, etc. C'est avec ça qu'on va faire, on sait. (Gabily, $2003: 104)$

Ce que Gabily souhaite interroger dans Gibiers du temps, comme dans une majeure partie de son œuvre, c'est donc la question du désir dont il dévoile le caractère monstrueux pour dire les rapports d'oppression qui s'exercent dans la société post-industrielle.

Voyons à présent comment l'analyse comparée entre plusieurs points fondamentaux du mythe tels que l'aveu de Phèdre ainsi que les conditions de sa mort, le penchant sauvage et chasseur d'Hippolyte et enfin le personnage de Thésée, offre la possibilité d'envisager l'évolution du rapport au désir confondu avec l'exercice du pouvoir. Il s'agit dès lors de faire appel aux héritages culturels afin d'envisager comment cette mémoire est travaillée dans le geste de création contemporain.

\section{L'aveu de Phèdre}

9 La version d'Euripide correspond en réalité à une seconde version du poète grec car une première version avait fait scandale comme le mentionne Marie Delcourt-Curvers dans son avant-propos de la pièce :

Euripide traita deux fois le sujet. Une première pièce fit scandale. Phèdre s'abandonnait à sa passion et la déclarait elle-même à son beau-fils qui, d'horreur, se voilait le visage. Ces audaces sont atténuées dans la pièce que nous lisons et qui, en avril 428, quelques mois après la mort de Périclès, obtint le premier prix. Phèdre et Hippolyte se rencontrent en scène, mais sans échanger ni un mot ni même un regard. Derrière le théâtre, la nourrice a fait la déclaration. En lui livrant son secret, Phèdre lui a en même temps donné licence d'agir, car sa résistance était liée à son mutisme. (Delcourt-Curvers, $1962:$ 201-202)

10 Ce qu'Euripide avait écrit dans un premier mouvement et que les règles morales de son temps ne lui ont pas permis de maintenir, ses successeurs l'ont mis en œuvre. Depuis Sénèque jusqu'à Ritsos et dans une évolution qui va de pair avec la morale dans laquelle s'inscrit chacun des auteurs, Phèdre dévoile son amour à Hippolyte sans que jamais cet aveu se fasse dans les coulisses du théâtre. Ritsos en fait même le sujet unique de son texte qui prend la forme d'un long monologue dans lequel Phèdre avoue son amour à un Hippolyte silencieux, interdit, et orchestre seule sa vengeance qui condamnera le jeune homme.

Chez Ritsos, Phèdre assume et revendique son désir, elle est une femme libérée de toute morale qui s'abandonne pleinement par le verbe à son impossible amant. Le texte de Ritsos n'est pas un texte théâtral mais un poème. C'est le poème de Phèdre qui déverse les mots et les maux de son désir. C'est le débordement de cette folie qui, dans les autres versions, occupe essentiellement les deux tirades dans lesquelles elle confie d'abord son amour pour Hippolyte à sa nourrice puis à Hippolyte lui-même. La place qu'accorde Ritsos 
à la parole de Phèdre déplace l'approche du mythe en libérant une parole qui était définie par l'obligation de contenir le désir comme les mots. Ici les mots coulent, inondent le destinataire silencieux. Phèdre tisse la toile qui enserre Hippolyte par ses mots quand lui ne peut que subir ce déversement et s'emmurer dans le silence. Elle est en scène jusqu'au bout et ses derniers mots sont la condamnation qu'elle annonce à Hippolyte :

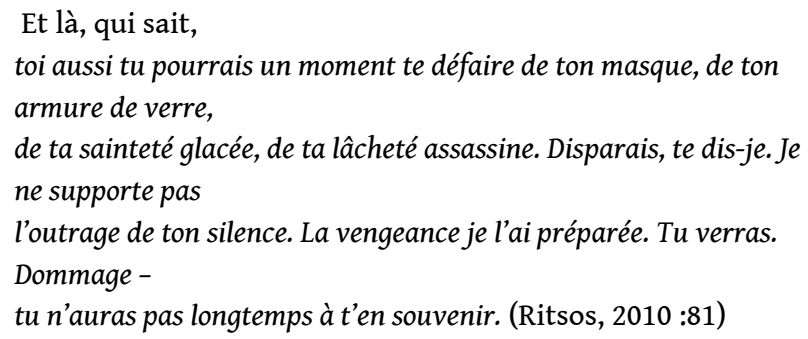

12 À cet endroit, Gabily se démarque de ses prédécesseurs car le sujet de sa pièce n'est pas dans le dévoilement du désir ni même dans la culpabilité ressentie par Phèdre. La Phèdre de Gabily n'a plus rien à avouer. Hippolyte est mort depuis longtemps ; elle lui a survécu et s'unit chaque année à un homme en mémoire de celui-ci, unions desquelles naissent uniquement des filles en signe d'une malédiction lancée par Hippolyte lorsqu'il mourut :

PHÈDRE. [...]

Vois-moi qui suis comme la montagne où souffle le désir des marâtres. Voici ce que je lui crie. Il ne répond pas, mon Hippolyte. Rien. Il me méprise. Je le vois bien, nourrice. Il se lève et il s'en va, le méprisant. Voilà. Je vois son jeune dos de méprisant. Je vois les muscles de son dos de jeune Dieu vaniteux qui ne veut rien réconcilier de Phèdre-moi ni de quiconque de mon sexe. Je vois la séparation, l'abîme que les dieux créèrent, la guerre depuis le commencement

Alors, meurs. Dis-je. Imbécile. Vaniteux. Egoïste. Minable

Ils ont lâché la meute sur lui, sur le bâtard, ils ont lâché les chiens de guerre de son père. Ah serviteurs, qu'avez-vous fait ? - Ce que tu nous as commandé, maîtresse - Il est mort en te maudissant - Il est mort disant qu'il te haïssait aussi de ce maudissement - Et que maintenant toute chose soit encore plus séparée, dit-il, de l'homme et de la femme, pour ce qui vient et te concerne, toi. - Que ta génération ne produise que des femelles lamentables, toi, dit-il, et ce n'est plus qu'un râle dans sa bouche : Zeus créateur, exauce-moi - Puis le chef de meute lui arrache la face, et il ne dit plus rien. (Gabily, 1995 : 44-45)

13 Cette tirade nous apprend plusieurs informations essentielles sur les points communs et les points de divergence qui existent entre les versions antérieures du mythe et celle de Gabily. On apprend ainsi que Phèdre a déclaré son amour à Hippolyte comme dans les autres versions mais que cette déclaration est antérieure au commencement de la fable. En cela, la fable hérite d'une situation dont elle décrit les conséquences selon la vision de l'auteur. On apprend aussi que ce n'est pas Thésée qui a ordonné la mort d'Hippolyte mais Phèdre; or, dans les versions antérieures, Phèdre ou la nourrice manipulaient Thésée par le mensonge, ce qui conduisait ce dernier à déclarer la mort de son fils. Chez Gabily, Thésée n'est jamais revenu dans sa maison ; il n'a donc pas pu condamner son fils. Phèdre est seule responsable de la mort d'Hippolyte qu'elle ordonna ; et elle devint criminelle par ce geste. Une criminalité qu'aucune justice ne vient sanctionner car le roi mythique qui hérite du pouvoir des dieux ${ }^{3}$ et a pouvoir de justice n'est pas revenu des enfers. Il intègre un élément dramaturgique supplémentaire : Phèdre n'enfantera désormais que des filles. Cette malédiction lancée par Hippolyte fait sens lorsque l'on sait que les femmes de la lignée de Pasiphaé sont maudites en amour et que c'est là un élément important du mythe de Phèdre. Cette variabilité permet à l'auteur d'introduire des personnages complémentaires : ce sont les filles nées de Phèdre et qui forment à leur tour un clan 
d'Amazones inspiré d'une situation réelle dans la banlieue de Los Angeles. Par cet ajout, l'auteur relie le mythe au réel en le réactualisant avec un combat féministe contemporain. Ces différents éléments, de proximité et d'écarts avec les textes antérieurs, placent la fable de Gabily dans un rapport de recréation et d'invention à partir des héritages. Dès lors, l'espace contemporain se regarde à l'aune des nombreux fracas provoqués par le choc des temporalités.

\section{La mort de Phèdre}

Chez Euripide, Ritsos et Tsvetaïeva, Phèdre se donne la mort par pendaison. Sénèque et Garnier choisissent l'épée d'Hippolyte et Racine le poison. De son côté Gabily reprend l'héritage d'Euripide mais en faisant de ce suicide une mascarade répétée à chaque anniversaire :

NOURICIELLE. Elle a encore essayé de se pendre. Mais j'avais préparé ce qu'il fallait PHÈDRE. Nourrice, j'ai froid. J'ai tombé. J'ai manqué. J'ai froid nourrice

DÉMOPHON. Enlevez-lui ces instruments de mort. Qu'on lui enlève ces instruments de mort, voyons.

PHÈDRE. Peux pas empêcher, non. Voudrais rester dans le lit de paresse et le lit de luxure mais le lit est comme le désert. Le fleuve des pensées charrie les glaces et les troncs et les berges arrachées. Les draps, les draps. Malheur. Malheur. Nuit. Nuit. Lève-toi, soleil qui réchauffe même plus.

Ébroue-toi, père brûlant.

Qu'est-ce que je vais faire. Comment que je vais continuer

HÉLÉNA. Comme toujours, maman

AGNA. Comme d'habitude, mère. La journée maudite va passer. (Gabily, 1995 :

16-17)

15 Au lieu d'une fin tragique, le désir de mort du personnage devient un ressort de la théâtralité et annule par la répétition de ce geste toute possibilité cathartique : ce n'est là que du théâtre. Ce jeu de dénonciation de la représentation qui se retrouve dans de nombreuses pièces gabilyennes neutralise la catharsis aristotélicienne. Dès lors la désactivation de l'acte censé inspirer la pitié implique une évolution dans le traitement du mythe mais aussi dans la fonction de la représentation théâtrale. Alors que, dans les versions antérieures, la pendaison de Phèdre vient précipiter l'action jusqu'à son dénouement, le jeu de répétition mis en place par Gabily avec le simulacre d'une pendaison rituelle et avortée inscrit la fable dans la continuité d'une histoire qui a commencé bien avant son ouverture. Cette conception non close de la dramaturgie participe du lien avec une histoire antérieure tout en ouvrant le drame à un temps qui dépasse celui du présent de la fable. Dans Gibiers du temps, Phèdre est donc restée dans l'espace des vivants et porte l'usure du temps:

CYPRIS. Elle ?/ ça ? / Phèdre ? / Qui fut de toute splendeur? / Qui fut ma rivale en beauté?

NOURRICIELLE. Je ne l'ai jamais connue belle mais je sais qu'elle a été belle - bien qu'il n'existe aucune photographie du temps de sa beauté. Sa beauté date de bien avant l'invention de la photographie. Les peintres même n'ont jamais fait son portrait dans sa beauté. Il paraît qu'il y avait des vases qui disaient sa beauté. On les a brisés. (Gabily, 1995 : 18)

Phèdre est restée prisonnière du temps. Elle est condamnée à l'éternité et au ressassement, à un manque viscéral qui rend son supplice insupportable, supplice qui n'est pas sans nous rappeler l'écho lointain de Sisyphe. Dès lors, elle réitère à chaque 
anniversaire de la mort d'Hippolyte le rituel de la pendaison ratée, ouvrant une journée qui s'achèvera par l'accouplement avec un homme, victime expiatoire offerte par ses fils :

NOURRICIELLE. A l'endroit où habituellement elle pose le tabouret. A l'endroit où le crochet est scellé depuis des siècles des siècles dans tous les palais qu'elle habita. A cet endroit où chaque année elle vient recommencer à se souvenir et à se vouloir pendre d'une faute que tous ont oubliée, je crois, je prépare la corde avec l'entaille dans la corde, jusqu'à la dernière torsade - elle-même bien entaillée. C'est ainsi que ma mère fit, et avant elle sa mère, et la mère de sa mère encore, avant-avant elle et pour le reste, je suis trop jeune et ignorante, je ne sais pas quel est cet homme que tu dis (Gabily, $1995: 18$ )

17 Parmi les emprunts qu'il est possible de déceler dans la fable gabilyenne, figure une approche christique du personnage d'Hippolyte que l'auteur a peut-être repris à Yannis Ritsos :

à mes doigts

s'accroche cette chaîne familière avec ta petite croix (celle

dont on dit que c'est la Déesse qui te l'offrit), celle

qui pendait à ta poitrine, qui s'embuait de la moiteur de ta chair ;

(oui c'est moi qui te l'ai volée)

[...]

Tu ne vois pas

ses empreintes, maillon après maillon, gravées dans ma chair ?-

et un petit Crucifié, taillé

entre mes seins, - j'imagine, que si tu l'embrassais

il ressusciterait réellement; encore que

je l'aie bien appris : la résurrection n'est

qu'acte solitaire du renoncement, et pas un acte de l'union. (Ritsos, $2010: 45-49$ )

Dans Gibiers du temps, cette parenté entre Hippolyte et le Christ s'exprime par le fait que la journée d'anniversaire qui commémore Hippolyte en consommant le corps d'un homme chassé par Acamas et Démophon, a lieu à chaque lundi de Pâques :

PHÈDRE. [...] C'est Vendredi de Pâques aujourd'hui, nourrice, t'en souviens-tu. Le jeune Seigneur va mourir. Et dans sa mort, je renaîtrai. Il vient, mon Hippolyte. Il va pousser la porte. Il viendra. Le jeune dieu avec sa croix d'amertume jusqu'à moi. Il gravira sa Phèdre, son Golgotha. (Gabily, 1995 : 44-45)

Vraisemblablement sous l'impulsion de Ritsos, Gabily intègre à sa fable une conception catholique du temps basée sur la résurrection: la consommation du corps de l'homme renvoyant à la consommation de l'hostie et assurant la résurrection de Phèdre et la perpétuation du crime. Dans ce mouvement, il relie son texte à celui du poète grec tout en inscrivant l'héritage religieux dans l'espace de sa fable. Cette dimension spirituelle produit deux dynamiques essentielles dans la pièce: d'une part la théâtralité de la répétition, d'autre part la transformation du rapport au divin dans l'histoire occidentale.

Dans la fable de Gabily, Hippolyte est donc totalement absent, il est le seul personnage du mythe qui le soit, ce qui distingue l'approche de Gabily des auteurs qui l'ont précédé. Cependant cette absence est fondamentale dans le fonctionnement dramaturgique de la pièce car elle irradie l'action: l'absence d'Hippolyte est la conséquence du crime de Phèdre et l'argument qui préside à la chasse à l'homme ritualisée. 


\section{La chasse : transposition}

21 Ce qui caractérise le personnage d'Hippolyte dans le mythe chez Euripide est la dévotion qu'il voue à la déesse de la chasse Artémis et la vie qu'il mène au milieu des bois parmi ses compagnons, fuyant toute présence féminine. C'est précisément ce mépris et cette haine à l'égard de la déesse Aphrodite qui est à l'origine de la passion coupable de Phèdre. Phèdre est la victime de la déesse qui l'utilise pour atteindre le fier et chaste Hippolyte. De toutes les versions abordées par Gabily, seule celle d'Euripide met en scène la déesse ; c'est par elle que s'ouvre la tragédie d'Euripide :

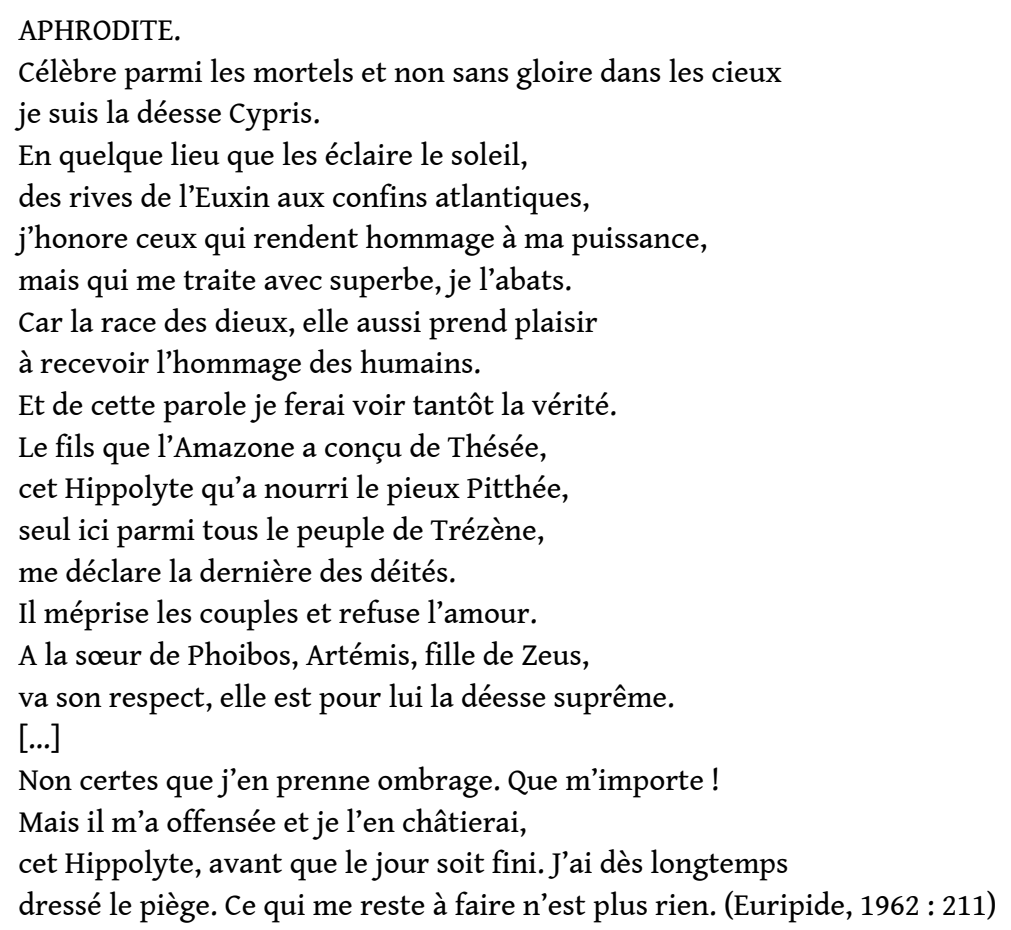
distribution des personnages et en lui attribuant la volonté d'orchestrer sa vengeance. Les paroles prononcées par la déesse à l'ouverture de Gibiers du temps, entrent en écho avec celles d'Euripide :

CYPRIS. (enlève sa barbe). Dis : Cypris sans Zeus / J'ai soufflé sur ses cendres. Dis : toujours aux avant-postes et ne renonçant pas.

Par ici,

nous ne sommes plus la majorité, comme tu peux le constater. Mes vieux camarades tombent comme des mouches, et tous les intermédiaires. (Pensive, désigne Hermès Archange, dit : ) Avec celui-là aussi j'ai dû coucher / etc. / avec qui n'ai-je pas couché / etc. (Songe encore. Semble chasser des images désagréables. Se reprend. Dit :) Là-bas-làhaut, malgré tout, ça peut encore aller.

Ouais. Pour Arès-armé, toujours, et pour moi, toujours,

à peu près. Ouais. Enfin, il me semble. Depuis le temps que je n'y suis pas retournée. Il faut que j'y retourne, je crois que j'ai oublié quelque chose qui te concernait.

Mais quoi. Quelque chose, oui

(Elle psalmodie sans conviction : ) «A vrai dire, je n'en suis pas jalouse / quel besoin en aurais-je / Mais du tort qu'il m'a fait/ je le punirai / Hippolyte / Tout ou presque est en chemin depuis longtemps / Je n'aurai pas grand-peine... (Gabily, 1995 : 14) 

rien et ne comprend pas les usages. Ainsi les fils sacrifieront leur père en offrande à leur mère. Le héros du mythe et vainqueur du Minotaure sera consommé par le Minotaurefait-femme de Gibiers du temps. Et alors que Thésée croit retrouver sa maison, son palais et sa famille après cette longue traversée dans la cité déréglée de la fable, il sera exécuté sur l'autel du nouvel ordre débarrassé des mythes. Ce gibier exceptionnel clôturera ainsi le cycle de répétition dans lequel Phèdre est enfermée et sa présence dérèglera la machine théâtrale qui existe en amont de la fable gabilyenne :

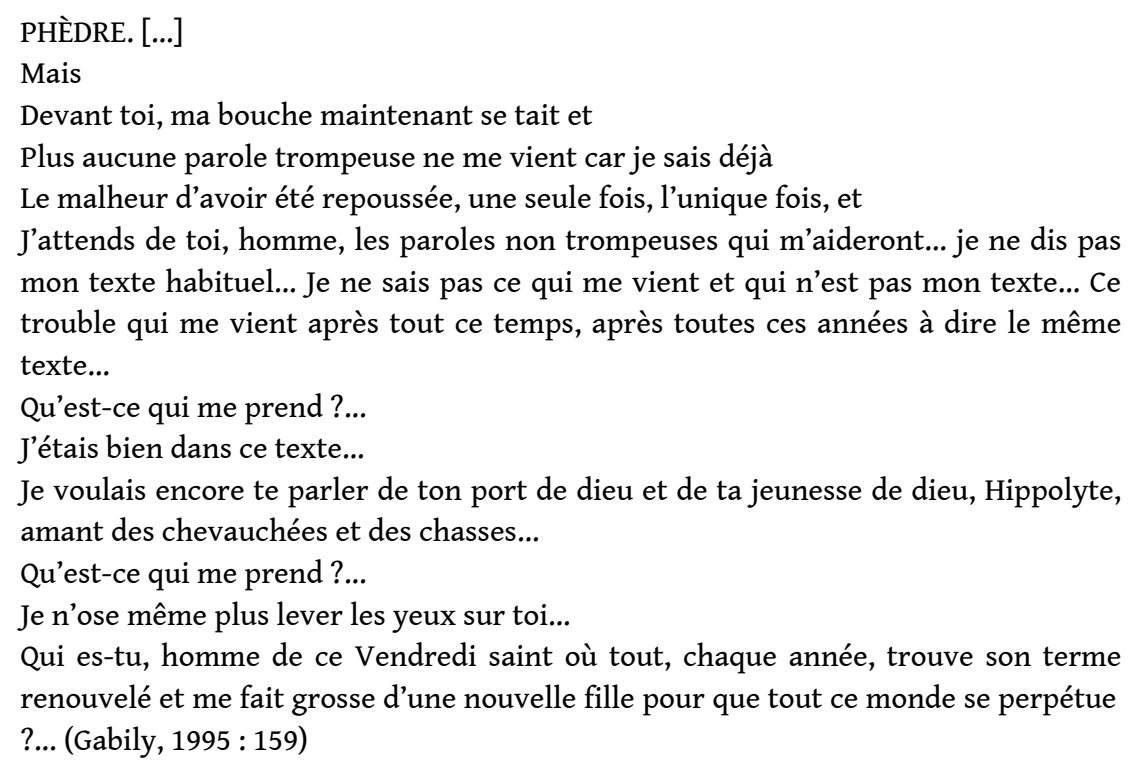

Comme pour le rituel de la pendaison, le sacrifice de la victime expiatoire est réglé selon des codes établis et répétés chaque année, rejoignant en cela les célébrations qui organisent le temps selon des cycles qui assurent l'équilibre entre les hommes et les dieux, entre les hommes et la nature. Dans la fable de Gibiers du temps, le cycle se rompt et 
vient clôturer un temps archaïque que la maladie de Phèdre maintenait au cœur de la ville contemporaine.

\section{Thésée : déchéance du héros}

Contrairement à ses prédécesseurs, Gabily fait intervenir Thésée dans l'action de sa pièce dès le début. Il va même jusqu'à déplacer la focale qui faisait de Phèdre le personnage central du mythe pour la diriger sur Thésée et sur le chemin de son retour vers la maison familiale. La fable de Gabily commence avec le retour de Thésée sur la terre, mais le temps passé aux Enfers a été plus long que dans toutes les autres versions du mythe: deux mille ans se sont écoulés depuis que ce dernier est parti de Trézène avec son compagnon Pirithoos dans le dessein d'enlever Perséphone, la femme d'Hadès :

Donc, Thésée [...] reviendrait des enfers. De nos jours. La scène se passe de nos jours. Thésée le fourbe, le malin, le corrupteur, le civilisateur, le violeur légitime, le législateur. Pour qui le sait. Un homme. Une sorte de héros. Ce siècle use tous les héros qui se présentent. [...] Le reste (la pièce) est ce qui traverse ceux [...] qui traverseront: des hommes, des femmes, encore, toujours, chasseurs et proies, grands fauves et petits gibiers, du temps et ceux qui le manipulent. Des acteurs, en somme. (Gabily, 2003 : 104-105)

Le temps de l'évolution et de l'adaptation à l'environnement sociétal contemporain lui a manqué. Le chemin de son retour sera fait de la traversée d'une société qu'il ne comprend pas et qui ne le comprend pas donnant ainsi à la fable une focale par laquelle la société contemporaine est regardée depuis celui qui n'en a pas les codes. Le regard de Thésée est celui qui dresse le procès de la société postmoderne qui a rompu avec les valeurs du passé et, plus largement, avec ses héritages. De tous les personnages présents dans la fable de Gabily, il est celui qui est le plus éloigné de la connaissance. Il est l'aveugle. Il est l'errant. Il est l'étranger. Celui par qui arrive le phénomène de rupture, la dislocation du sens. $\mathrm{Ne}$ comprenant pas cette société dans laquelle il est revenu, il est le gibier du temps. Le décalage qui réside entre les tenants de la société antique et ceux de la société libérale contemporaine est à même de produire une interrogation sur l'évolution de la société humaine. Celui qui croit avoir été délivré des Enfers pour revenir dans son pays se retrouve dans un autre enfer qu'est l'étrangeté au monde :

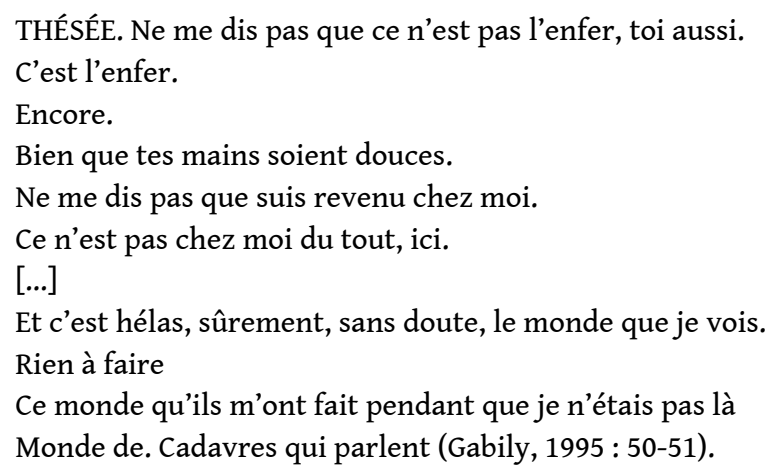

La figure du héros grec disparait au profit de celle de l'étranger nécessairement voué à l'errance dès lors que ses repères socioculturels ne sont plus valides. Le personnage de Thésée cristallise le choc des civilisations et introduit en cela notre rapport à l'héritage de même qu'à l'altérité. Sommes-nous étrangers à nous-mêmes dès lors que nous détruisons les legs du passé ? Ici le legs n'est pas anodin car l'étranger massacré à la fin du triptyque porte, entre autres attributs mythiques, le symbole de la fondation d'Athènes et du 
système démocratique grec. C'est aussi celui par qui arrive la constitution de classes entre les hommes d'une cité :

Après la mort d'Égée, Thésée, débarrassé des Pallantides, prit le pouvoir en Attique. Son premier acte fut de réaliser le "synœcisme", c'est-à-dire de réunir en une seule cité les habitants jusque-là disséminés dans la campagne. Athènes fut la capitale de l'Etat ainsi constitué. Il la dota des bâtiments politiques essentiels : le Prytanée, la Boule, etc. Il institua la fête des Panathénées, symbole de l'unité politique de l'Attique. Il battit monnaie, divisa la société en trois classes : les Nobles, les Artisans et les Cultivateurs, et instaura dans ses grandes lignes, le fonctionnement de la démocratie, telle qu'elle existait à l'époque classique. (Grimal, $1994: 453)$

Y a-t-il encore de la place pour quiconque ne souscrirait pas à la loi d'un libéralisme qui se nourrit du commerce des corps ? C'est là une des questions posées par Gibiers du temps. Dans cette pièce, les victimes sont celles d'une guerre qui prend le nom du libéralisme : les corps sont des matières premières faisant l'objet de tractations entre les personnages ; c'est notamment le cas du corps de Thésée que les Amazones et les fils de Phèdre se disputent. Comme pour toutes les guerres, celle-ci fait des victimes : les plus défavorisés, les plus démunis qui ne prennent pas part aux lois du commerce qui s'est étendu à toutes les relations humaines, ainsi qu'en témoigne cette note de travail de Gabily :

Commerce (des hommes-femmes entre eux), assouvissement (des hommes-femmes entre eux), commerce (qui achète qui ? qui profite de qui ? quelle guerre profitable se joue ailleurs, à quel profit ?), faillite (dont on se remet, dont on ne se remet pas selon la place où l'on se tient), etc. (Gabily, $2003: 104)$

31 La pièce se termine par le massacre de la famille mythique sanctionnée par un personnage portant le nom de Sanguier, homme de mains d'Acamas et Démophon. Une fois l'héritage liquidé, il instaure un nouvel ordre et c'est l'entrée dans l'ère de l'oubli où l'on se détourne des victimes de l'histoire pour construire un nouveau projet de société :

SANGUIER. Tu n'avais pas demandé à voir ces ruisseaux de sang. Je suis l'homme nouveau. Je suis le retour aux premiers temps. C'est moi. Je n'aime pas comme tu ne me fais plus confiance. Ferme les yeux et tu les verras plus, ces ruisseaux de sang. Aide-moi à me laver et je serai de nouveau dans la pureté. Blanc, comme de l'argent blanchi et comme l'oubli qui nous domine. (Gabily, 1995 : 173-174)

Mais à l'oubli réclamé par l'homme nouveau qui veut se débarrasser de sa mémoire et du souvenir de tous les morts, Gabily oppose la voix mémorielle de la Pythie, seule survivante des temps mythiques qui, depuis la scène d'un peep-show, prend en charge le récit des mémoires et renonce ainsi, après sa traversée dans la ville contemporaine, à dire l'avenir :

PYTHIE. [...] Elle fit. Rien qu'une main avec l'eau et le linge qui contient l'eau, et qui est l'oubli. Est-ce que tu peux comprendre

Tu peux comprendre, je sais. Homme, tu peux comprendre. L'eau qui efface et le linge qui la contient, et qui est l'oubli

Et tu jettes l'eau. Le linge, tu le presses. Tu peux comprendre, homme. Ainsi tes blessures sont effacées, et les morts qui peuplent ton chemin sont effacés, et ta mort

$[\ldots]$

Le Dieu m'a traversée qui était mon amour, puis le Dieu ne m'a plus fait signe

[...]

Je ne vagirai plus pour lui l'histoire future des hommes, et ce fut ma semence et ma peine que de vagir l'histoire des hommes et leur peine

$[\ldots]$

Veux-tu ma disparition, aussi 
Car je dis les noms de tous ceux qui fondèrent et de tous ceux qui disparurent. Je dis les noms

Car c'est mon lent travail de dire les noms (Gabily, 1995 : 174-175).

La Pythie de Gabily inscrit la mémoire du passé plutôt que la prophétie d'un futur car le Dieu qui était son amour ne lui fait plus signe. Une façon pour l'auteur de dire que l'avenir s'est dissous et qu'il ne reste du passé qu'un champ de ruines et de cadavres qui soufflant dans les ailes d'un ange effaré.

\section{米}

$\mathrm{Au}$ terme de notre traversée dans les invariants mythiques interrogés à travers les personnages constitutifs du mythe dans l'histoire du théâtre, nous pouvons mesurer combien le traitement contemporain réalisé par Didier-Georges Gabily inscrit son geste de création dans un héritage théâtral tout en révélant les ruptures du temps et en intégrant une réflexion sur la société post-moderne. Les changements opérés par Gabily sont radicaux : il propose des personnages contraires à ce qu'ils ont toujours été dans les versions antérieures, y compris celle plus récente de Ritsos qui resserre l'action sur l'aveu de Phèdre à Hippolyte. Mais la différence majeure qui réside entre le mythe tel qu'il est réinvesti dans Gibiers du temps et les différentes adaptations auxquelles il a été confronté réside dans le fait que l'auteur décale son écriture dans une temporalité différente de celle de ses prédécesseurs : Gabily n'écrit pas tant une adaptation du mythe aux valeurs de la société contemporaine qu'une suite aux versions antérieures auxquelles le dénouement aurait été enlevé. Gibiers du temps peut être vu comme la fin du mythe dans l'espace contemporain. Il fait entrer une dimension du temps qui dépasse les règles antérieures du vraisemblable en chargeant les personnages d'une éternité languissante. Dès lors il ne serait pas incongru de proposer l'hypothèse que la liquidation finale de tous les personnages mythiques - à l'exception de la Pythie - est à même d'évoquer la liquidation de ce temps immémorial auquel le mythe fait appel.

\section{BIBLIOGRAPHIE}

ARISTOTE (1996). Poétique. Paris : Gallimard.

DELCOURT-CURVERS, Marie (1962). « Avant-propos », in EURIPIDE, Tragédies complètes I. Paris :

Gallimard, pp. 201-202.

EURIPIDE (1962). Tragédies complètes I. Paris : Gallimard.

GABILY, Didier-Georges (1994). Chimère et autres bestioles. Arles : Actes Sud -Papiers.

GABILY, Didier-Georges (1995). Gibiers du temps. Arles : Actes Sud -Papiers.

GABILY, Didier-Georges (1996). TDM3 - Théâtre du Mépris 3. Arles : Actes Sud -Papiers.

GABILY, Didier-Georges (2003). Notes de travail. Arles : Actes Sud. 
GABILY, Didier-Georges (2008). Contention, in GABILY, Didier-Georges. CEuvres. Arles : Actes Sud Papiers.

GARNIER, Robert (1923). CEuvres complètes I. Paris : Librairie Garnier.

GENETTE, Gérard (1982). Palimpsestes. Paris : Éditions du Seuil.

GRIMAL, Pierre (1994). Dictionnaire de la mythologie grecque et romaine. Paris : Presses Universitaires de France.

HOMÈRE (1955). Odyssée. Paris : Gallimard.

LEROY, Séverine (2015). L'œuvre théâtrale de Didier-Georges Gabily : poétique d'une mémoire en pièces. HAL Archives-ouvertes.fr [on-line]. <URL : https://hal.archives-ouvertes.fr/tel-01151125>.

LÉVI-STRAUSS, Claude (1997). Anthropologie structurale deux. Paris : Pocket.

MORAVIA, Alberto (1989). Le Mépris. Paris : Flammarion.

RACINE, Paul (1971). Phèdre. Paris : Librairie Larousse.

RICGUR Paul (2000). La mémoire, l'histoire, l'oubli. Paris : Seuil.

RITSOS, Yannis (2010). Phaidra. Cassaniouze : ÉrosOnyx Éditions.

SÉNÈQUE (2012). Théâtre complet. Arles : Actes Sud.

\section{NOTES}

1. Nom donné au groupe d'acteurs formés dans les ateliers de D.G.- Gabily et qui sera le nom de la compagnie professionnelle dans laquelle seront créées les mises en scènes à partir de 1991.

2. Tous les auteurs ayant été travaillés pendant le stage ont écrit une version du mythe pour le théâtre à l'exception de Ritsos qui en a plutôt fait un long poème, un long monologue mais qui a souvent été porté à la scène.

3. Dans le mythe, Thésée condamne Hippolyte non par les moyens de la justice humaine mais en invoquant la promesse de son père Poséidon d'exaucer trois vœux formulés par Thésée.

\section{RÉSUMÉS}

Cette étude aborde les dynamiques de la réécriture du mythe de Phèdre et d'Hippolyte dans le travail théâtral de Didier-Georges Gabily (1955-1996). Elle est développée selon une approche génétique puis par le prisme d'une analyse comparée avec des versions théâtrales antérieures (Euripide, Sénèque, Garnier, Racine, Ristos). À travers le jeu de transposition et d'hypertextualité, la pièce Gibiers du temps interroge l'espace contemporain dans la perspective d'une déconstruction post-moderne.

This study approaches dynamic rewriting of the myth of Phèdre and Hippolyte in the theatrical work of Didier-Georges Gabily (1955-1996). It is developed in a genetic approach then in an analysis compared with former theatrical versions (Euripide, Sénèque, Garnier, Racine, Ristos). 
Through the game of transposition and hypertextuality, the play Gibiers du temps questions contemporary space from the point of view of the post-modern deconstruction.

INDEX

Keywords : Gabily (Didier-Georges), contemporary dramaturgy, hypertextuality, myth, Phèdre Mots-clés : Gabily (Didier-Georges), dramaturgie contemporaine, hyper-textualité, mythe

\section{AUTEUR}

\section{SÉVERINE LEROY}

Université Rennes 2

severine.leroy.rennes[at]gmail.com 